\title{
Estresse salino em duas variedades de cana-de-açúcar: enzimas do sistema antioxidativo e fluorescência da clorofila ${ }^{1}$
}

\author{
Salinity stress in two varieties of sugar cane: enzymes of the antioxidant system and \\ chlorophyll fluorescence
}

\author{
Lilia Willadino ${ }^{2 *}$, Ronaldo Alves de Oliveira Filho ${ }^{3}$, Elias Arcanjo da Silva Junior ${ }^{4}$, Artur Gouveia Neto e $^{5}$ \\ Terezinha Rangel Camara ${ }^{6}$
}

\begin{abstract}
Resumo - O Brasil destaca-se por ser o maior produtor de cana-de-açúcar (Saccharum officinarum L.) e por dominar todos os estágios da tecnologia de produção de açúcar e etanol. Atualmente se observa a expansão dessa cultura para a região semiárida brasileira, a qual apresenta solos propensos à salinização. O presente trabalho objetivou avaliar a resposta à salinidade de duas variedades de cana-de-açúcar. O ensaio foi implantado em casa de vegetação, em delineamento inteiramente casualizado, em esquema fatorial 2 x 8 com cinco repetições, correspondendo a duas variedades de cana-de-açúcar (RB867515 e RB863129) e oito níveis de $\mathrm{NaCl}(0 ; 25 ; 50 ; 75 ; 100 ; 125 ; 150 ; 200 \mathrm{mM}$ de $\mathrm{NaCl})$. A salinidade induziu aumento na fluorescência e diminuição na concentração de clorofila em ambas as variedades. A redução da clorofila, entretanto, foi maior na variedade RB867515. Observou-se, nesta mesma variedade, aumento da atividade da peroxidase do ascorbato (APX) e da catalase (CAT), enquanto na variedade RB863129 ocorreu redução na atividade dessas enzimas. Esses resultados indicam que a variedade RB867515 apresenta um mecanismo mais eficiente na proteção da planta contra danos no aparato fotoquímico e contra a ação de espécies reativas de oxigênio, em particular $\mathrm{o}_{2} \mathrm{O}_{2}$.
\end{abstract}

Palavras-chave - Catalase. Fluorescência da clorofila. Peroxidase. Saccharum officinarum.

\begin{abstract}
Brazil is seen as being the largest producer of sugarcane (Saccharum officinarum L.) and of mastering all stages in the production of sugar and ethanol. Currently this production is being expanded into the semi-arid regions of Brazil, where there are soil-types prone to salinity. This study aimed to evaluate the response to salinity in two sugarcane genotypes, RB867515 and RB863129. Salinity caused an increase in chlorophyll fluorescence and a reduction of chlorophyll concentration in both varieties. The reduction of chlorophyll however was higher in RB867515. In this same type, an increase in the activity of ascorbate peroxidase (APX) and catalase (CAT) was observed, while in genotype RB863129 there was a reduction in the activity of these two enzymes. These results show that RB867515 has a mechanism which better protects the plant against damage to the photochemical system and the action of reactive oxygen species, particularly $\mathrm{H}_{2} \mathrm{O}_{2}$.
\end{abstract}

Key words - Chlorophyll fluorescence. Peroxidase. Catalase. Saccharum officinarum.

\footnotetext{
* Autor para correspondência

${ }^{1}$ Recebido para publicação em 31/08/2010; aprovado em 31/01/2011

Trabalho submetido e selecionado no primeiro Simpósio Brasileiro de Salinidade realizado de 12-15/10/2010 em Fortaleza, Ceará, Brasil; Pesquisa financiada pela CAPES e CNPq

${ }^{2}$ Departamento de Biologia/UFRPE, Rua dom Manoel de Medeiros s/n, Recife-PE, Brasil, 52.511-900; lilia@pq.cnpq.br

${ }^{3}$ Graduando do curso de Agronomia/UFRPE, Rua dom Manoel de Medeiros s/n, Recife-PE, Brasil, 52.511-900

${ }^{4}$ Programa de Pós-Graduação em Física Aplicada/UFRPE, Rua dom Manoel de Medeiros s/n, Recife-PE, Brasil, 52.511-900

${ }^{5}$ Departamento de Física/UFRPE, Rua dom Manoel de Medeiros s/n, Recife-PE, Brasil, 52.511-900

${ }^{6}$ Departamento de Química/UFRPE, Rua dom Manoel de Medeiros s/n, Recife-PE, Brasil, 52.511-900
} 


\section{Introdução}

As áreas de cultivo de cana-de-açúcar têm se expandido para a região semiárida brasileira. Esta região caracteriza-se por apresentar condições edafoclimáticas favoráveis à salinização dos solos. Os efeitos da salinidade sobre as plantas incluem restrição na assimilação do $\mathrm{CO}_{2}$ e queda no teor de clorofila (SILVA et al., 2010), resultando, frequentemente, na redução do crescimento da planta (MUNNS et al., 2002). Todo estresse ambiental pode acarretar estresse oxidativo, resultado do acúmulo de espécies reativas e citotóxicas de oxigênio $\left(\mathrm{O}_{2}{ }^{--}, \mathrm{H}_{2} \mathrm{O}_{2}, \mathrm{OH}\right)$ (MITTLER, 2002).

As plantas possuem um mecanismo de defesa antioxidativo que atua mediante a ativação de um sistema enzimático (AGARWAL; PANDEI, 2004) e que inclui a peroxidase do ascorbato (APX), a catalase (CAT) e a peroxidase (POD). Essas enzimas catalisam a decomposição do $\mathrm{H}_{2} \mathrm{O}_{2}$, desempenhando um papel fundamental no equilíbrio redox (MITTLER, 2002).

É frequente o aumento da atividade de uma ou mais enzimas do sistema antioxidativo, em função do incremento da salinidade, como registrado para a APX (BOR et al. 2003; KOCA et al., 2007; MAIA et al., 2010), CAT (ABOGADALLAH et al, 2010; AGARWAL; PANDEI, 2004; KOCA et al., 2007) e POD (ABOGADALLAH et al., 2010). O aumento da atividade dessas enzimas minimiza os efeitos deletérios do $\mathrm{H}_{2} \mathrm{O}_{2}$ sobre a célula e suas estruturas (MAIA, 2002).

Este trabalho teve como objetivo avaliar a resposta de duas variedades de cana-de-açúcar ao estresse salino, mediante a variação da atividade das enzimas do sistema antioxidativo (CAT, POD, APX), da fluorescência e do teor de clorofila.

\section{Material e métodos}

O experimento foi conduzido em casa de vegetação da Universidade Federal Rural de Pernambuco - UFRPE, em Recife - PE. Foram utilizadas duas variedades de cana-de-açúcar, RB863129 e RB867515. As mudas foram procedentes do Centro de Tecnologia Estratégicas do Nordeste (CETENE), em Recife - PE. Ambas as variedades foram submetidas a oito níveis de salinidade $(0 ; 25 ; 50 ; 75 ; 100 ; 125 ; 150 ; 200 \mathrm{mM}$ de $\mathrm{NaCl})$ em esquema fatorial $2 \times 8$, inteiramente casualizado. Foram mantidas cinco repetições por tratamento. Trinta dias após a diferenciação do experimento, a massa fresca das plantas foi determinada por meio de balança de precisão (Kern410) e as plantas congeladas em nitrogênio líquido para análises bioquímicas. Foi avaliada a atividade de enzimas do sistema antioxidativo, o teor de clorofila, e a fluorescência induzida por laser. Para obtenção do extrato enzimático, macerou-se $0,2 \mathrm{~g}$ de massa fresca em nitrogênio líquido. As atividades enzimáticas foram expressas em unidade enzimática. Uma unidade da enzima é definida como a quantidade de enzima que causa um aumento de 0,001 unidade de absorbância por minuto e expressas como U $\mathrm{min}^{-1} \mathrm{mg}^{-1}$ de proteína solúvel.

A atividade da catalase foi determinada pela adição de $100 \mu \mathrm{L}$ do extrato enzimático a 2,9 mL de uma solução contendo $\mathrm{H}_{2} \mathrm{O}_{2}(12,5 \mathrm{mM})$ e tampão fosfato de potássio $(50 \mathrm{mM})$, com $\mathrm{pH} 7,0$. A absorbância foi medida a $240 \mathrm{~nm}$, usando espectrofotômetro UV-VIS (HAVIR; MCHALE, 1987). Para a determinação da atividade da peroxidase foram adicionados $100 \mu \mathrm{L}$ do extrato enzimático bruto a $0,9 \mathrm{~mL}$ da mistura de reação contendo tampão fosfato de potássio $25 \mathrm{mM}$, pH 6,8, acrescida de pirogalol a $20 \mathrm{mM}$ e $\mathrm{H}_{2} \mathrm{O}_{2}$. a $20 \mathrm{mM}$. A absorbância, medida a $420 \mathrm{~nm}$, foi obtida 60 segundos após o início da reação (KAR; MISHRA, 1976). A atividade da peroxidase do ascorbato foi determinada mediante a adição de $100 \mu \mathrm{L}$ do extrato ao meio de reação constituído por $2,7 \mathrm{~mL}$ de tampão fosfato de potássio a $50 \mathrm{mM}, \mathrm{pH} 6,0$, e $75 \mu \mathrm{L}$ de ácido Lascórbico 0,8 mM. A absorbância foi medida a $290 \mathrm{~nm}$ (NAKANO; ASADA, 1981, modificado por KOSHIBA, 1993). A determinação do teor de clorofila seguiu o método de Arnon (1949): o tecido vegetal foi macerado, utilizando-se acetona a $80 \%$, e centrifugado por cinco minutos a $2000 \mathrm{~g}$. A absorbância foi medida a $663 \mathrm{~nm}$ e $645 \mathrm{~nm}$.

As medidas de fluorescência da clorofila foram realizadas utilizando-se uma fonte de excitação no UV, azul, verde, vermelho ou infravermelho associada a um sistema de coleta de sinal de fluorescência por cabos de fibras ópticas, acoplado a um espectrômetro com fotodetector. Os dados foram tratados e analisados usando o software Ocean Optics USB HR4000.

Os dados obtidos foram submetidos à análise de variância pelo teste ' $F$ ' e regressão. Para o fator “dose de salinidade" realizou-se análise de regressão, por ser um fator quantitativo. Para o fator "variedade", por ser qualitativo, as médias foram comparadas pelo teste de Tukey, a 5\% de probabilidade, utilizando-se o programa estatístico ASSISTAT (SILVA; AZEVEDO, 2002).

\section{Resultados e discussão}

Ambas as variedades apresentaram redução na biomassa fresca da parte aérea em função do incremento dos níveis de sal na solução nutritiva (FIG. 1), entretanto, destaca-se o melhor desempenho da variedade RB867515 (TAB. 1). O efeito deletério da salinidade sobre o 
Tabela 1 - Resumo da análise de variância para peso de massa fresca (MF), fluorescência da clorofila (F690/F735), teor de clorofila, atividade da peroxidase do ascorbato (APX), catalase (CAT) e peroxidase (POD) de duas variedades de cana-de-açúcar aos 30 dias de tratamento em distintos níveis de salinidade

\begin{tabular}{ccccccc}
\hline \multirow{2}{*}{ Fator de Variação } & MF & F690/F735 & clorofila & APX & CAT & POD \\
\hline Variedade (V) & $953,37^{* *}$ & $0,03^{* *}$ & $0,01^{*}$ & $3.955 .805,08^{* *}$ & $146.810,66^{*}$ & $31.978 .924,98^{* *}$ \\
Nível de sal (S) & $994,12^{* *}$ & $0,80^{* *}$ & $4,28^{* *}$ & $1.829 .748,12^{* *}$ & $243.692,37^{\text {ns }}$ & $37.340 .726,63^{* *}$ \\
Interação (VxS) & $125,47^{* *}$ & $0,07^{* *}$ & $0,42^{* *}$ & $2.429 .862,82^{* *}$ & $397.009,30^{\text {ns }}$ & $6.067 .688,18^{* *}$ \\
\hline Tratamentos & $586,04^{* *}$ & $0,41^{* *}$ & $2,19^{* *}$ & $2.251 .538,78^{* *}$ & $308.781,49^{\text {ns }}$ & $22.389 .188,57^{* *}$ \\
Resíduo & 22,05 & 0,002 & 0,06 & $406.818,63$ & $271.409,29$ & $9.665,72$ \\
\hline CV \% & 23,35 & 2,26 & 18,39 & 28,76 & 26,23 & 2,47 \\
\hline
\end{tabular}

** significativo a $1 \%$, e * significativo a $5 \%$ (teste $\mathrm{F}$ ); ns não significativo

crescimento da planta já está bem documentado e, frequentemente, em uma mesma espécie, a cultivar sensível apresenta maior redução do crescimento do que a tolerante (MUNNS et al., 2002; KOCA et al., 2007; CORREIA et al., 2009).

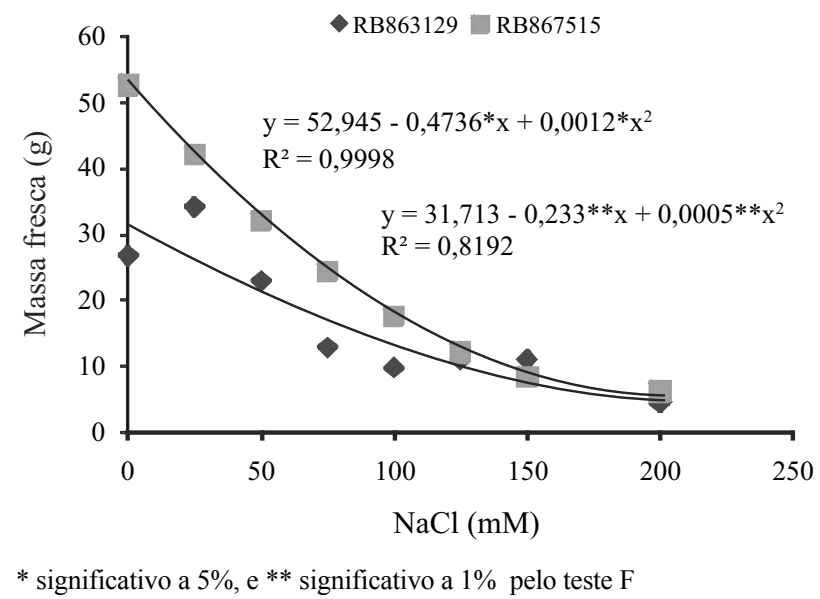

Figura 1 - Massa fresca de parte aérea de cana-de-açúcar, variedades RB 867515 e RB 863129, submetidas a distintos níveis de $\mathrm{NaCl}$ durante 30 dias

A redução de produção de massa fresca, à medida que ocorreu um incremento dos níveis de sal na solução, foi acompanhada pelo aumento na fluorescência das moléculas de clorofila (FIG. 2 e 3), indicando que houve perturbação no processo fotossintético. Em condições de estresse a taxa de fluorescência (FR) das bandas de emissão da clorofila no vermelho e vermelho distante, denotadas pela relação F690/F735, é aumentada (SCHREIBER,
1983; STOBER; LICHTENTHALER, 1992). O aumento dessa relação F690/F735 indica que a capacidade de captação da luz é superior à capacidade de aproveitamento fotoquímico, resultando na emissão de fluorescência.

Paralelamente ao aumento da fluorescência observouse um decréscimo no teor de clorofila. $O$ coeficiente de correlação negativa entre essas duas variáveis foi altamente significativa tanto na variedade RB863129 (-0,847) como na variedade RB867515 (-0,854). A variedade RB867515,

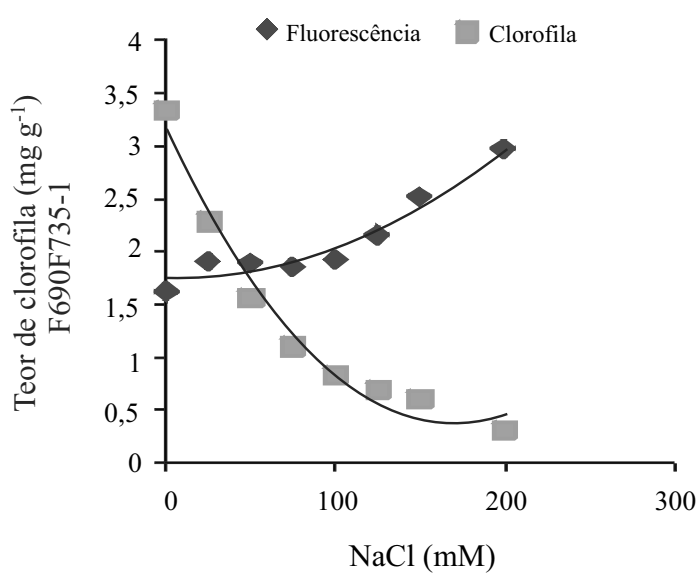

$$
\begin{array}{ll}
\mathrm{y}=1,7375-0,0003^{* *} \mathrm{x}+3 \mathrm{E}-05^{* *} \mathrm{x}^{2} & \mathrm{R}^{2}=0,9474 \\
\mathrm{y}=3,1467-0,033^{* *} \mathrm{x}+1 \mathrm{E}-04 * * \mathrm{x}^{2} & \mathrm{R}^{2}=0,9755
\end{array}
$$

** significativo a $1 \%$ pelo teste $\mathrm{F}$

Figura 2 - Teor de clorofila em $\mathrm{mg} \mathrm{g}^{-1}$ massa fresca $(\square)$ e fluorescência de clorofila F690/F735 ( $\diamond$ ) da parte aérea da variedade RB867515 submetida durante 30 dias a distintos níveis de $\mathrm{NaCl}$ 


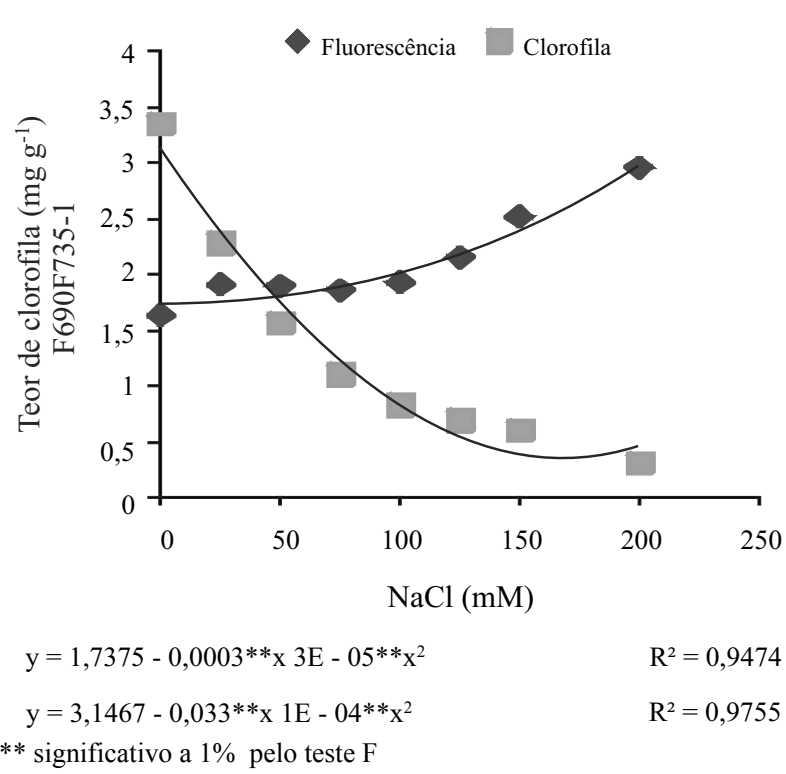

Figura 3 - Teor de clorofila em $\mathrm{mg} / \mathrm{g}$ massa fresca e fluorescência de clorofila F690/F735 da parte aérea da variedade RB863129 submetida durante 30 dias a distintos níveis de $\mathrm{NaCl}$

entretanto, apresentou um maior incremento na fluorescência e uma maior redução no teor de clorofila do que a variedade RB863129 (TAB. 1). A variedade RB867515, portanto, apresenta uma maior aclimatação ao estresse imposto, visto que a maior redução do teor de clorofila é um mecanismo de defesa por reduzir a captação de energia luminosa e consequentemente diminuir o fluxo de elétrons para a cadeia de transferência de elétrons, uma vez que essa está altamente reduzida favorecendo a redução parcial do oxigênio que resulta na formação de espécies reativas de oxigênio (ROS) (BROSCHÉ et al., 2010). A redução do teor de clorofila em função do incremento do nível de sal é uma resposta frequente em várias espécies vegetais, entre elas Grewia tenax (HUNSCHE et al., 2010), Jatropha curcas (SILVA et al., 2010) e Tamarindus indica (HUNSCHE et al., 2010). É interessante destacar que algumas espécies, como o Arachis hipogaea, não apresentam alteração significativa nos parâmetros associados à emissão da fluorescência sob condições de estresse (CORREIA et al., 2009).

A condição de desequilíbrio entre a atividade fotoquímica e bioquímica da fotossíntese favorece a geração de ROS (SOUZA et al., 2005), o que resulta numa maior demanda por um eficiente mecanismo antioxidante de defesa para evitar o estresse oxidativo (CAVALCANTI et. al., 2004). No que se refere à decomposição de espécies reativas, e mais especificamente do $\mathrm{H}_{2} \mathrm{O}_{2}$, observou-se, de uma maneira geral, o incremento na atividade da APX e da CAT em ambas as variedades quando submetidas a até aproximadamente $100 \mathrm{mM}$ de $\mathrm{NaCl}$. A partir de $100 \mathrm{mM}$ de $\mathrm{NaCl}$, estresse salino severo, observou-se uma queda progressiva nas atividades dessas enzimas (FIG. 4 e 5).

As variedades de cana-de-açúcar RB867515 e RB863129 apresentaram valores similares da atividade da peroxidase do ascorbato (APX) no tratamento controle e no de $25 \mathrm{mM}$ de $\mathrm{NaCl}$ (FIG. 4). No entanto, os valores de atividade divergem entre as duas variedades em função do aumento da concentração salina. $\mathrm{Na}$ variedade RB863129 a atividade da APX decaiu em aproximadamente $190 \mathrm{U} \mathrm{min} \mathrm{mg}^{-1}$ de proteína solúvel, a cada incremento de $25 \mathrm{mM}$ de $\mathrm{NaCl}$. Por outro lado, na variedade RB867515 ocorreu um incremento da atividade dessa enzima, com valores máximos atingidos a 75 e $100 \mathrm{mM}$ de $\mathrm{NaCl}$. As APXs são consideradas as enzimas mais importantes na eliminação de $\mathrm{H}_{2} \mathrm{O}_{2}$ tanto no citosol quanto nos cloroplastos (INZÉ; VAN MONTAGU, 1995). Geralmente o maior incremento na atividade da APX caracteriza variedades com maior tolerância ao $\mathrm{NaCl}$ como constatado em Sesamum indicum (KOCA et al., 2007), Beta vulgaris (BOR et al. 2003 ), Lycopersicon (MITTOVA et al. 2002), e Vigna unguiculata (MAIA et al., 2010). A partir de $100 \mathrm{mM}$ de $\mathrm{NaCl}$ observou-se o decréscimo da atividade da APX na variedade RB 867515. Segundo Munns et al. (2002) níveis de $\mathrm{NaCl}$ superiores a $100 \mathrm{mM}$ provocam a inibição de uma vasta gama de enzimas.

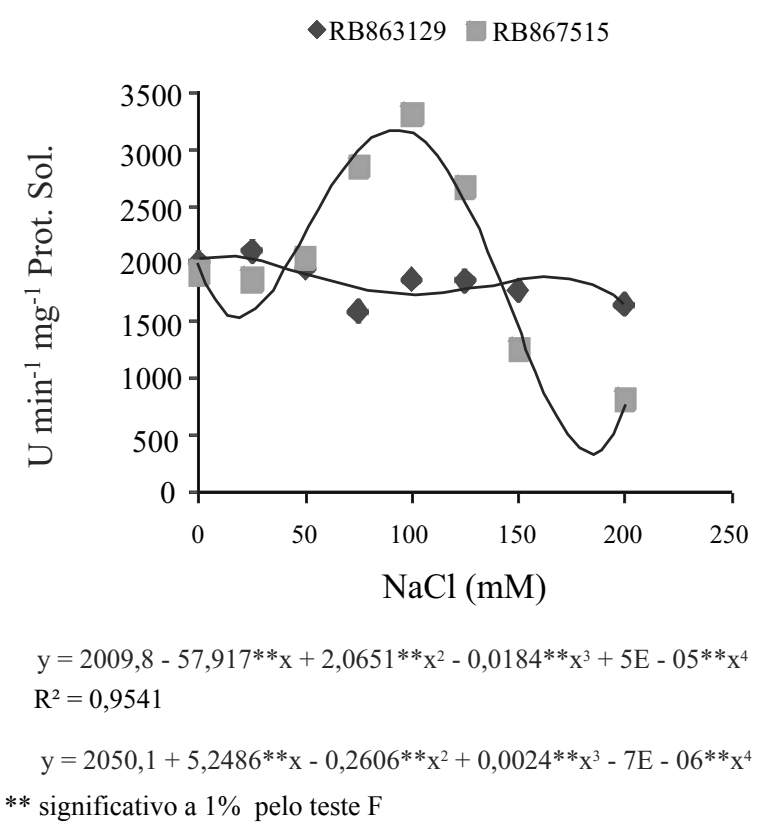

Figura 4 - Atividade da enzima, peroxidase do ascorbato (APX) (EC 1.11.1.11) da parte aérea de duas variedades de cana-deaçúcar submetidas durante 30 dias a distintos níveis de $\mathrm{NaCl}$ 
Da mesma forma que a APX, a atividade da CAT foi maior na variedade RB867515 (TAB. 1, FIG. 5). Em diversas espécies como Sesamum indicum (KOCA et al., 2007), Cassia angustifolia (AGARWAL, PANDEI, 2004), Echinochloa crusgalli L. (ABOGADALLAH et al, 2010) e Chenopodium álbum (YAO et al., 2010) foi observado um incremento da atividade da CAT, sendo o mesmo considerado uma habilidade para fazer frente às espécies reativas de oxigênio. $\mathrm{Na}$ variedade RB863129, foi observado decréscimo moderado da atividade da CAT quando as plantas foram submetidas ao estresse salino.

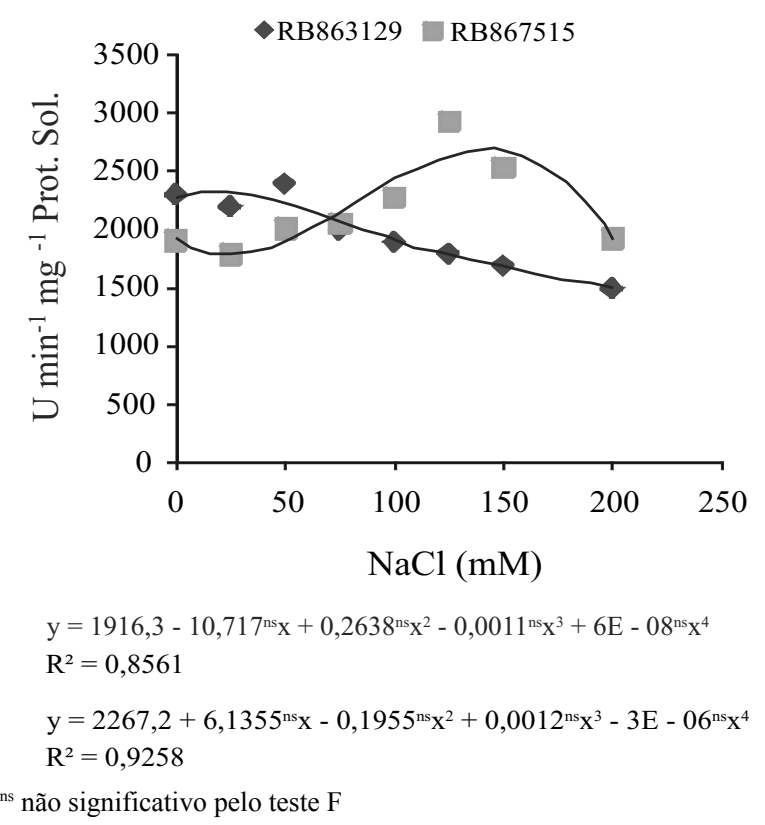

Figura 5 - Atividade da enzima catalase (CAT) (EC 1.11.1.6) da parte aérea de duas variedades de cana-de-açúcar submetidas durante 30 dias a distintos níveis de $\mathrm{NaCl}$

A atividade da POD, na variedade RB867515, tendeu a se manter constante até a concentração de $100 \mathrm{mM} \mathrm{de} \mathrm{NaCl}$, a partir da qual detectou-se o incremento da atividade dessa enzima (FIG. 6). A variedade RB863129, por sua vez, também apresentou tendência de aumento da atividade da POD a partir de $100 \mathrm{mM}$ de $\mathrm{NaCl}$. O aumento da atividade da peroxidase minimiza os efeitos prejudiciais do estresse salino e, ademais, responde pela regulação fina da concentração do $\mathrm{H}_{2} \mathrm{O}_{2}$ em função da grande afinidade com esta ROS (MITTLER, 2002; ABOGADALLAH et al., 2010).

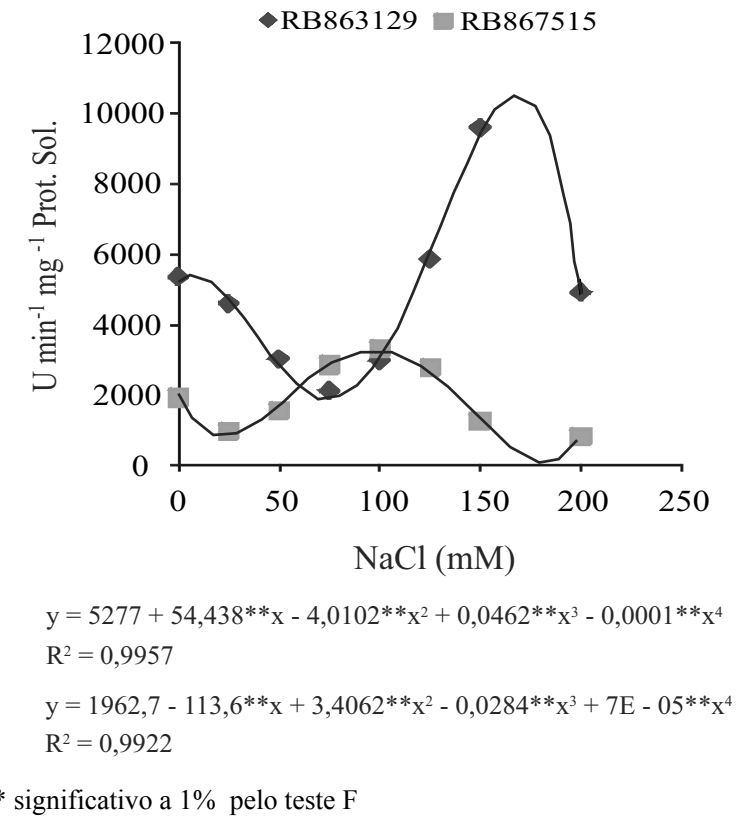

Figura 6 - Atividade da enzima peroxidase (POD) (EC 1.11.1.7) da parte aérea de duas variedades de cana-de-açúcar submetidas durante 30 dias a distintos níveis de $\mathrm{NaCl}$

Os resultados indicam a maior capacidade de aclimatação da variedade RB867515, uma vez que apresentou uma menor redução da massa fresca associada ao maior incremento da atividade da peroxidase do ascorbato (APX) e da catalase (CAT), além de redução do teor de clorofila, caracterizando sua estratégia de aclimatação.

\section{Conclusão}

A relação entre a maior atividade enzimática e o maior crescimento indica uma melhor aclimatação da variedade RB867515 quando comparada à variedade RB863129 sob estresse salino, na fase inicial de crescimento. Observa-se, em ambas as variedades, um aumento na fluorescência paralelo à redução do teor de clorofila à medida que ocorre incremento dos níveis de sal, indicando a perturbação no processo fotossintético.

\section{Referências}

ABOGADALLAH, G. M.; SERAG, M. M.; QUICK, P. W. Fine and coarse regulation of reactive oxygen species in the salt tolerant mutants of barnyard grass and their wild-type parents under salt stress. Physiologia Plantarum, v. 138, n. 01, p. 60-73, 2010. 
AGARWAL, S.; PANDEY, V. Antioxidant enzyme responses to $\mathrm{NaCl}$ stress in Cassia angustifoli. Biologia Plantarum, v. 48, n. 04, p. 555-560, 2004.

ARNON, D. I. Copper enzymes in isolated chloroplasts: polyphenoloxydase in Beta vulgaris. Plant Physiology, v. 24, n. 01, p. 1-15, 1949.

BOR, M.; OZDEMIR, F.; TURKAN, I. The effect of salt stress on lipid peroxidation and antioxidants in leaves of sugar beet Beta vulgaris L. and wild beet Beta maritima L. Plant Science, n. 164, n. 01, p. 77-84, 2003.

BROSCHÉ, M. et al. Stress Signaling III: Reactive Oxygen Species (ROS). In: PAREEK, A. et al. Abiotic Stress Adaptation in Plants: Phisiological, Molecular and Genomic Foundation. Dordrecht: Springer, 2010. Cap. 5, p. 91-102.

CAVALCANTI, F. R. et al. Superoxide dismutase, catalase and peroxidase activities do not confer protection against oxidative damage in salt-stressed cowpea leaves. New Phytologist, n. 163, n. 03, p. $563-71,2004$.

CORREIA, K. G. et al. Crescimento, produção e características de fluorescência da clorofila a em amendoim sob condições de salinidade. Revista Ciência Agronomica, v. 40, n. 04, p. 514-521, 2009.

HAVIR, E. A.; MCHALE, N. A. Biochemical and developmentl characterization of multiple forms of catalase in tabaco leaves. Plant Physiology, v. 84, n. 2, p. 450-455, 1987.

HUNSCHE, M. et al. Effects of $\mathrm{NaCl}$ on surface properties, chlorophyll fluorescence and light remission, and cellular compounds of Grewia tenax (Forssk.) Fiori and Tamarindus indica L. leaves. Plant Growth Regulation, v. 61, n. 03, p. 253-263, 2010.

INZÉ. D.; VAN MONTAGU, M. Oxidative stress in plants. Current Opninion in Biotechnology, v.6, n. 02, p 153-158, 1995.

KAR M.; MISHRA D. Catalase, peroxidase and polyphenoloxidase activities during rice leaf senencence. Plant Physiology, v. 57, n. 02, p. 315- 319, 1976.

KOCA, H. et al. The effect of salt stress on lipid peroxidation, antioxidative enzymes and proline content of sesame cultivars. Environmental and Experimental Botany, v. 60, n. 03, p. 344-351, 2007.
KOSHIBA, T. Cytosolic ascorbate peroxidase in seedlings and leaves of maize (Zea mays). Plant and Cell Physiology, v. 34, n. 05, p. 713-721, 1993.

MAIA, J. M. et al. Antioxidative enzymatic protection in leaves of two contrasting cowpea cultivars under salinity. Biologia Plantarum, v. 54, n. 01, p. 159-163, 2010.

MITTLER, R. Oxidative stress, antioxidants and stress tolerance. Trends in Plant Science, v. 7, n. 09, p. 405-410, 2002.

MITTOVA, V. et al. Salt stress induces upregulation of an efficient chloroplast antioxidant system in the salt-tolerant wild tomato species Lycopersicon pennellii but not in the cultivated species. Physiologia Plantarum, v. 115, n. 03, p. 393-400, 2002.

MUNNS, R. et al. Avenues for increasing salt tolerance of crops, and the role of physiologically based selections traits. Plant Soil, v. 247 , n. 01 , p. $93-105.2002$.

NAKANO Y.; ASADA K. Hydrogen peroxide is scavenged by ascorbate-especific peroxidase en spinach chloroplasts. Plant Cell Physiology, v. 22, n. 05, p.867-880, 1981.

SCHREIBER, U. Chlorophyll fluorescence as a tool in plant physiology. 1: The measuring system. Photosynthesis Research, v. 4, n. 04, p. 361-375, 1983.

SILVA, F. A. S.; AZEVEDO, C. A. V. Versão do programa computacional Assistat para o sistema operacional Windows. Revista Brasileira de Produtos Agroindustriais, v. 4, n. 01, p. 71-78, 2002.

SILVA, E. N. et al. Comparative effects of salinity and water stress on photosynthesis, water relations and growth of Jatropha curcas plants. Journal of Arid Environments, v. 74, n. 10, p. 1130-1137, 2010.

SOUZA, R. P. et al. Photosynthetic responses of young cashew plants to varying environmental conditions. Pesquisa Agropecuária Brasileira, v. 40, n. 08, p.735-744, 2005.

STOBER, F.; LICHTENTHALER, H. K. Change of laser-induced blue, green, and red fluorescence signature during greening of etiolated leaves of wheat. Journal of Plant. Physiology, v. 140, n. 06, p. 673-680, 1992.

YAO, S. et al. Plant growth and responses of antioxidants of Chenopodium album to long-term $\mathrm{NaCl}$ and $\mathrm{KCl}$ stress. Plant Growth Regul, v. 60, n. 02, p. 115-125, 2010. 